\title{
Investigación bibliométrica de los trabajos de grado sobre liderazgo en las facultades de psicología de la ciudad de Bogotá (Colombia)
}

Bibliometric Research of Degree Papers on Leadership in Psychology Schools in the City of Bogotá, (Colombia)

Pesquisa bibliométrica das teses e dissertações sobre liderança nas faculdades de psicologia da cidade de Bogotá (Colômbia)

\author{
Mónica García Rubiano* \\ María Constanza Aguilar Bustamante ${ }^{\star \star}$ \\ Estefanía Payan Wilson ${ }^{* \star *}$ \\ Diego Mauricio Forero Duran ${ }^{* * * *}$
}

Fecha de recibido: 12 de febrero de 2014

Fecha de aprobado: 17 de septiembre de 2014

Doi: dx.doi.org/10.12804/rev.univ.empresa.28.2015.07

* Magíster en Psicología, Especialista en Psicología de las Organizaciones y Psicóloga de la Universidad Católica de Colombia (Bogotá, Colombia). Docente-Invesigadora de la Universidad Santo Tomás (Bogotá, Colombia). Correo electrónico: monica.garcia@gmail.com

** Estudiante de doctorado en Psicología de la Universidad de la Laguna (Santa Cruz de Tenerife, España), Magíster en Psicología de la Universidad Católica de Colombia (Bogotá, Colombia), Especialista en Psicología del Consumidor de la Fundación Universitaria Konrad Lorenz (Bogotá, Colombia), Especialista en Educación de la Universidad El Bosque (Bogotá, Colombia), Psicóloga de la Universidad Católica de Colombia (Bogotá, Colombia). Miembro del Consejo General de Colegios Oficiales de Psicólogos. Correo electrónico: mariaaguilar@usantotomas.edu.co

*** Psicóloga de la Universidad Santo Tomas (Bogotá, Colombia). Analista de Selección en Claro Colombia. Correo electrónico: stephicol16@yahoo.com.mx

**** Psicólogo de la Universidad Santo Tomas (Bogotá, Colombia). Correo electrónico: diegoforeroduran@yahoo.com.co 
Para citar este artículo: García Rubiano, M., Aguilar Bustamante, M. C., Payan Wilson, E., \& Forero Duran, D. M. (2015). Investigación bibliométrica de los trabajos de grado sobre liderazgo en las facultades de psicología de la ciudad de Bogotá (Colombia). Universidad \& Empresa, 17(28), 147-172. Doi: dx.doi.org/10.12804/ rev.univ.empresa.28.2015.07

\section{RESUMEN}

Esta investigación hace un análisis bibliométrico de los trabajos de grado sobre liderazgo, entre 2000 y 2011, en las facultades de psicología adscritas a la Asociación Colombiana de Facultades de Psicología (Ascofapsi) en la ciudad de Bogotá. El muestreo usado es intencional o de conveniencia. El estudio es de tipo descriptivo. En él se realizan 1) un análisis desde la estadística descriptiva en el que se encuentra la frecuencia de los datos obtenidos y 2) un análisis lexicométrico de datos textuales que fue llevado a cabo con la ayuda del software SPAD ${ }^{\circledR}$.

Palabras clave: análisis bibliométrico, análisis lexicométrico, Bogotá, liderazgo, trabajos de grado en psicología.

\section{ABSTRACT}

This bibliometric research analyzes degree papers on leadership, between 2000 and 2011, in the psychology schools members of the Colombian Association of Psychology Schools (Ascofapsi) in the city of Bogota. The sampling used was intentional or by convenience. The study is descriptive. In it, 1) an analysis based on the descriptive statistics in which the frequency of the data obtained is found and 2) a lexicometric analysis of textual data that was carried out with the help of SPAD ${ }^{\circledR}$ software, were made.

Keywords: Bibliometric analysis, lexicometric analysis, Bogota, leadership, degree papers on psychology.

\section{RESUMO}

Esta pesquisa faz uma análise bibliométrica das teses e dissertações sobre liderança, entre os anos 2000 e 2011, nas faculdades de psicologia adscritas à Associação Colombiana de Faculdades de Psicologia (Ascofapsi) na cidade de Bogotá. A amostragem utilizada é intencional ou de conveniência. O estudo é de tipo descritivo. Nele realizam-se 1) uma análise desde a estatística descritiva no que se encontra a frequência dos dados obtidos e 2) uma análise lexicométrica de dados textuais que foi levado a cabo com a ajuda do software SPAD ${ }^{\circledR}$.

Palavras-chave: análise bibliométrica, análise lexicométrica, Bogotá, liderança, teses e dissertações em psicologia. 
1. INTRODUCCIÓN Y REVISIÓN DE LA LITERATURA

\subsection{Liderazgo}

El liderazgo es tal vez la característica positiva que más desea desarrollar el ser humano. Quien la posee suele tener dominio y aceptación dentro de un grupo al momento de realizar acciones y movilizar a los demás para que logren un cometido específico (Shein, 2001).

Frente a este tema se han podido desarrollar distintas investigaciones que logran despertar en las personas habilidades propicias para el manejo de los grupos (Capowski, 1994; Cendrós, Rincón, \& Ugas, 2004; Helland, Wazeter, \& Bayazit, 2009). Autores como Eden (1992) y Benatuil y Castro (2007) han logrado señalar habilidades, actitudes y características que debe desarrollar un líder para alcanzar resultados idóneos en el trabajo que está desempeñando.

El liderazgo se refiere a la influencia que ejerce una persona sobre su grupo de referencia. A través de esta, el líder logra que otros lleven a cabo las tareas que se pretenden desarrollar al servicio de la eficacia colectiva (Fleishman et al., 1991).

Partiendo de lo anterior, se concluye que el líder es la persona que ejerce el mencionado dominio de acuerdo a unos patrones que guían a su equipo, para el desarrollo de tareas específicas que están dirigidas por él mismo. Aunque este las coordine, es su grupo quien logra desarrollar de manera asertiva las tareas asignadas. Lo hace alcanzando los resultados que han sido promovidos por el líder y realizados a través del trabajo conjunto de los miembros del grupo (Hollander \& Julian, 1969; House \& Podsakoff, 1994; Yukl, 2008).

Pero el liderazgo no es solo el poder que ejerce una persona para lograr el desarrollo de tareas, es también el proceso por el cual un individuo tiene o no la capacidad para motivar a sus seguidores, haciendo que estos contribuyan al logro de los objetivos establecidos para un determinado proyecto organizacional (House, Javidan, Honses, \& Dorfman, 2002; Mendoza, Ortiz, \& Parker,2007;Yukl \& Van Fleet, 1992). El liderazgo incorpora una serie de variables, las cuales hacen que el líder logre su ejecución de la mejor manera posible. Este, por ejemplo, debe ser una persona asertiva al momento de motivar a sus seguidores. Esta característica, operada sobre ellos, consigue mejores resultados y suele hacerlo en tiempos más cortos (Eagly \& Karau, 2002; Mendoza \& Ortiz, 2006; Paglis \& Green, 2002).

El líder no es entonces solo aquel individuo que tiene influencia sobre 
los otros, ni quien posee el control absoluto de una situación; el buen líder es aquel que sabe leer o interpretar bien los contextos para ejecutar su labor de manera adecuada y no perder el control o el respeto de sus subordinados, es alguien que sabe amoldarse a su grupo para posicionarse positivamente dentro de él. De hecho, no solo el líder influye en el grupo, este también lo hace, de manera recíproca, sobre él. Así, en el juego de estas relaciones, se crean ambientes de trabajo favorables orientados al logro de resultados (Sánchez, Tapia, \& Becerra, 2006).

Ahora bien, aunque el líder ejerce un nivel de mando o coordinación dentro de un grupo, el liderazgo, como un todo, depende de diversos fenómenos sociales que se dan en la interacción entre los integrantes de un equipo. Este trata, de manera conjunta, de realizar ciertas actividades orientadas a la resolución de problemas y a la consecución de propósitos comunes entre la organización y la sociedad (Mendoza, 2008).

Estos aspectos son importantes. Ellos implican considerar las características y habilidades que requiere desarrollar un líder para manejar un grupo, así como para operar en el contexto en el que este se encuentra inmerso (Eden, 1992; Eisenbach,
Watson, \& Pillai, 1999; Hater \& Bass, 1988). La cultura aparece entonces como un aspecto significativo cuya consideración debe hacer parte del desarrollo de un líder. Así, por ejemplo, el lugar donde él y su grupo se encuentren debe ser manejado cautelosamente para no afectar creencias y comportamientos de su grupo de trabajo. Por esta razón la forma de ejercer liderazgo varía dependiendo, entre otros aspectos, del género (masculino o femenino) y de la cultura. De este modo, el liderazgo que tienden a ejercer las mujeres se caracteriza por ser más conectivo e interactivo y por estar enfocado a generar redes, nutrir las relaciones y empoderar a las personas (Bass \& Avolio, 1994; Godoy \& Mladinic, 2009).

Con base en las perspectivas y la complejidad del tema en mención, Muñoz (1986 citado en García, 2012), agrupó las distintas definiciones de liderazgo en cinco grandes categorías, a saber: 1) aquellas que conciben al líder como la persona central de un grupo, el miembro que más comunicaciones emite y recibe y que constituye el punto focal del mismo. Este se define además como aquel a quien los demás miembros del grupo quieren asemejarse y con el cual se identifican de algún modo; 2) las que conciben al líder como la persona preferida de un grupo, aquella con la cual la 
mayoría desea asociarse a la hora de realizar una determinada tarea; 3) las definiciones que señalan al líder como el miembro más capaz de un grupo para conducirlo hacia sus objetivos, desarrollando la estructura de interacción necesaria para resolver un problema común; 4) aquellas que lo conciben como el jefe institucional, quien ostenta el cargo de director de un grupo, según dictan las estructuras sociales establecidas, y 5) las que indican que lo esencial en el líder es su capacidad de influencia sobre el resto de los miembros del grupo. Esta última es la postura que se asume en este trabajo de investigación.

\subsection{Modelos de liderazgo}

Los modelos del liderazgo son una variable importante dentro del direccionamiento del líder, debido a que este tiene efectos directos sobre las decisiones y los resultados (Pedraja, Rodríguez, \& Rodríguez, 2006). Los modelos de liderazgo son uno de los temas más amplios en este campo de estudio. Estos incorporan una serie de argumentos útiles para guiar a los líderes respecto a cómo deben exaltar individual y socialmente características esenciales para determinar las habilidades del grupo.

El liderazgo transformacional, en particular, es definido por Bass, Avo- lio, Jung y Berson (2003), como la manera en que el líder motiva a sus seguidores, siendo este sincero y directo en relación con lo que espera de ellos y la manera como pueden incrementar sus resultados. El líder requiere persuadir a sus seguidores generando en ellos proactividad y un mayor impacto de las labores que realizan, así como lograr la coordinación de su trabajo hacia la consecución de intereses comunes en el equipo (Bass, 1999; Bass \& Avolio, 2000). Este estilo de liderazgo es común entre los líderes que predominan en las organizaciones, ya que son ellos quienes posicionan a la organización según la visión, la misión y los valores por los cuales esta se rige (Hinkin \& Tracey, 1994; House \& Shamir, 1993; Massi \& Cooke, 2000).

El liderazgo transaccional, por su parte, se define como la orientación del líder hacia las metas establecidas, las cuales están regidas por un mecanismo de incentivos y recompensas, que se otorgan una vez los objetivos trazados se ven cumplidos. A diferencia del liderazgo transformacional, en este el subordinado solo se limita a cumplir con las condiciones estipuladas con el fin de recibir algo a cambio (Naranjo, 2001). Frente a lo anterior, es importante señalar la continua retroalimentación que se le da al grupo de trabajo por parte 
del líder en cuanto a la gestión que se está realizando. Esto hace que se cree una independencia marcada entre los equipos de trabajo y el líder (Mendoza, Ortiz, \& Parker, 2007).

Aunque estos dos modelos de liderazgo siguen siendo vigentes, $y$ han logrado dar resultados a quienes los utilizan, algunas investigaciones (Bass \& Avolio, 1992; Bass, Avolio, Jung, \& Berson, 2003) han encontrado que los modelos transaccional y transformacional han evolucionado. Esto ha generado que sobresalga una dimensión más, que antes no había sido percibida. Esta dimensión es reconocida como el liderazgo del tipo laissez-faire. Se considera este modelo debido a que dentro de los estudios realizados existen líderes que no son asertivos en la toma de decisiones, formando o aumentando una dimensión negativa que permita describir un modelo pertinente para este tipo de situaciones que suelen presentarse en algunos de ellos.

Otro modelo interesante en el estudio del liderazgo es el de 'Vroom'. Este logra determinar la participación que se debe ejercer según la decisión que se deba tomar. Está basado en la motivación, la expectativa y la calidad de la información (Cardona, 2004). En este estilo lo más importante es la participación que debe alcanzar el grupo con su líder, es decir, debe existir entre los dos acompañamiento y participación directa, dos actitudes que les permitan llegar a conseguir soluciones de manera más rápida y exacta. En este caso el líder no solo direcciona a su grupo hacia un objetivo en particular, deben ser uno y otro, de manera conjunta, los que van direccionados hacia el mismo objetivo, haciendo que el líder sea una persona propositiva y acompañe al subordinado en las labores que realiza (Cardona 2004).

\subsection{Teorías del liderazgo}

La teoría de los rasgos se destaca por resaltar las características mentales, físicas y personales que debe crear el líder para generar entre su grupo un estilo de liderazgo exitoso (Naranjo, 2001). El objetivo principal de esta teoría es hacer que el líder paute en él ciertas características que lo hagan legítimo en la coordinación de su grupo y estas, al mismo tiempo, influyan en la manera en la que este ejerce el liderazgo.

Otra teoría que también ha sido importante dentro de los estudios del liderazgo es la de contingencia (House, Wright, \& Aditya, 1997). Esta argumenta que la manera en como el líder dirige depende de sus propias características, las cuales actúan según el ambiente, los subordinados y las habilidades que ellos poseen, 
con el fin de obtener resultados óptimos que dependan de las variables y factores mencionados. Frente a lo anterior cabe destacar que, aunque el líder es la persona que decide manejar la situación dependiendo de las variables mencionadas, es él quién debe aclarar el camino por el cual su equipo se debe guiar para alcanzar sus objetivos (Naranjo, 2001).

Por otra parte, la teoría del liderazgo situacional es definida por Sánchez (2000) como la capacidad que tiene el líder para diagnosticar las condiciones del contexto en las cuales se encuentra inmerso y poder realizar una combinación adecuada acerca de los tipos de conducta que se deben manejar según la situación.

En relación con las teorías mencionadas es importante concluir que el liderazgo ha logrado adquirir importancia gracias a múltiples planteamientos que han generado impacto y dinamismo en los líderes y sus grupos, logrando que ellos se preparen no solo en lo intelectual, sino también en lo personal y ambiental. Esto para poder ejecutar una coordinación acorde a un estilo que considere propio y que pueda adoptar para generar un mayor impacto.

\subsection{Estilos de liderazgo}

Los estilos de liderazgo se describen como la forma en la que un líder adopta de manera adecuada un sistema que le permita encaminar a su equipo hacia la consecución de las metas propuestas. Pedraja y Rodríguez (2004) destacan los siguientes: liderazgo participativo, liderazgo colaborativo y liderazgo instrumental.

Antes de darle continuidad a la definición de estas tres categorías, es importante aclarar la diferencia que existe entre los modelos y los estilos de liderazgo. Los primeros logran mostrarle y darle la oportunidad al líder de enfocarse y escoger la forma adecuada que según él encamina a sus colaboradores hacia la consecución de las tareas asignadas; los segundos, le permiten al líder utilizarlos en cualquiera de las situaciones que este considere convenientes para la ejecución de las tareas.

El estilo de liderazgo participativo, en primer lugar, se define como la consideración que tiene el líder de las opiniones que brinda su equipo de trabajo y el sentido que cobran para este las decisiones que se toman. El líder le informa a sus seguidores las decisiones que se van a tomar y los hace participes de su ejecución (Yukl, 1989).

El estilo de liderazgo colaborativo, en segundo lugar, se caracteriza por la ayuda que le brinda el líder a su equipo de trabajo y la atención que 
le puede proporcionar en pro de su bienestar y del seguimiento de este hacia los resultados (Yukl \& Van Fleet, 1982). Es decir, este no solamente le brinda a su equipo las herramientas necesarias para la realización de su trabajo, sino que también hace parte del desarrollo de sus ideas y la manera en como estas se van encaminando.

Por último, en el estilo de liderazgo instrumental el líder se encarga de brindar a sus subordinados la información necesaria para dejar claros algunos aspectos claves para la buena ejecución de su trabajo. En esta perspectiva es trascendental que el líder deje todos los aspectos claros para la resolución de problemas, ya que este no está tan dispuesto a la aclaración de las dudas frecuentes que pueda tener su equipo (Pedraja \& Rodríguez, 2004).

\subsection{Estudios bibliométricos}

Según Bordons y Zulueta (1999) los estudios bibliométricos tienen por objeto el tratamiento y análisis cuantitativo de las publicaciones científicas. A través de estos se busca complementar las opiniones y juicios que son emitidos por expertos. Proporcionan entonces herramientas útiles y objetivas en los procesos de evaluación de los resultados de la actividad científica.
Cruz y Javela (2004), por su parte, plantean que los estudios blibliométricos permiten el análisis de aspectos cuantitativos con el fin de evaluar diferentes productos científicos y campos de conocimiento. Estosconstituyen una herramienta clave para evidenciar y consolidar la información sobre objetivos, teorías, conceptos y métodos que orientan el quehacer de las disciplinas en las diferentes temáticas de estudio. Sirven también para señalar los logros de un determinado campo del conocimiento, así como proveer indicadores que permitan establecer el grado de desarrollo de las disciplinas científicas y el nivel de contribución al conocimiento del ser humano.

El presente estudio, en línea con lo indicado, contribuye a identificar la producción de trabajos de grado realizada entre 2000 y 2011 acerca del liderazgo. Esto con el fin de reconocer teorías, metodologías utilizadas, poblaciones objeto de estudio, características y variables de los instrumentos y técnicas de evaluación. Lo anterior permite determinar, desde los indicadores bibliométricos definidos para el estudio, el estado de la investigación en el tema y establecer un referente para formular a futuro proyectos de investigación en el campo. Permite también hacer la revisión y consolidación de modelos teóricos que permitan abordar la temática desde diferentes perspectivas. 
La pregunta de investigación que fundamenta el trabajo es: ¿cuál ha sido la producción de los trabajos de grado sobre liderazgo en las facultades de psicología adscritas a Ascofapsi entre 2000 y 2011 ?

Los objetivos formulados son:

1. Objetivo general: identificar las tendencias que ha tenido la producción de los trabajos de grado realizados sobre liderazgo en las facultades de psicología de la ciudad de Bogotá adscritas a ASCOFAPSI (Asociación Colombiana de Facultades de Psicología) entre 2000 y 2011.

2. Objetivos especificos:

a. Describir los indicadores bibliométricos de las investigaciones sobre liderazgo, entre 2000 y 2011, de los trabajos de pregrado y posgrado de las facultades de psicología de la cuidad de Bogotá adscritas a Ascofapsi.

b. Describir los indicadores lexicométricos arrojados por la información dada por el programa SPAD $^{\circledR}$ sobre las investigaciones realizadas en liderazgo entre 2000 y 2011 de los trabajos de pregrado y posgrado de las facultades de psicología de la cuidad de Bogotá adscritas a Ascofapsi.

\section{METODOLOGÍA}

\subsection{Diseño}

Esta investigación es de tipo descriptivo, es decir, que detalla los hechos y características de una situación o población y permite tanto la caracterización de la muestra tomada como de las variables que con mayor frecuencia se encuentran en el estudio (Hernández, Fernández, \& Baptista, 2006).

Dentro del estudio se realizan dos tipos de análisis, uno de carácter descriptivo bibliométrico y otro de datos textuales. Según Camps (2007), el análisis descriptivo bibliométrico consiste en revisar, resumir, identificar y sistematizar toda la información obtenida a través de la revisión que se realizó de los trabajos de grado de las facultades de psicología de las universidades adscritas a Ascofapsi entre 2000 y 2011. El análisis de datos textuales, por su parte, brinda la posibilidad de organizar, describir, resumir y comparar las diferentes temáticas entre los periodos determinados sobre el tema de liderazgo, para el estudio abordado en los trabajos de grado de pregrado y posgrado (Aguilar, López, Barreto, Rey, \& Vargas, 2007; Barreto, Borja, Sabucedo, López \& Alzate, 2010; Lebart, Salem, \& Bécue, 2000). 


\subsection{Participantes}

Para efectos de esta investigación se tuvo en cuenta a las universidades de Bogotá que se encuentran adscritas a Ascofapsi, así como los trabajos de grado relacionados con la temática de investigación. Esto generó un total de 22 unidades de análisis.

\subsection{Instrumento}

Se adaptó un formato de registro en donde serelacionólainformación de los indicadores. Para la recolección se utilizó como medio tecnológico el programa Excel, el cual contenía veinte variables consideradas pertinentes para este estudio. A continuación, se relacionan las variables bibliométricas que fueron adaptadas desde Meneses, Calle y García (2008):

1. Número y porcentaje de trabajos de grado por año.

2. Título de cada trabajo de grado.

3. Resumen de cada trabajo de grado.

4. Objetivo general de cada trabajo de grado.

5. Objetivos específicos de cada trabajo de grado.

6. Pregunta de investigación de cada trabajo de grado.

7. Número y porcentaje de palabras claves de los trabajos de grado.

8. Número y porcentaje de trabajos de grado de acuerdo con la afiliación de los autores.
9. Distribución de trabajos de grado por número de firmas.

10. Autores más citados en cada uno de los trabajos de grado.

11. Número y porcentaje de trabajos de grado de acuerdo al tipo de documento.

12.Definición sobre liderazgo de cada trabajo de grado.

13. Variables incluidas en cada trabajo de grado.

14.Distribución de los trabajos de grado según la temática relacionada en cada uno.

15.Distribución de los trabajos de grado según el enfoque de investigación.

16. Distribución de los trabajos de grado de acuerdo con el tipo de estudio.

17.Número y porcentaje de los instrumentos utilizados, para hacer la recolección de la información en los estudios.

18. Número y porcentaje de tesis por número de participantes que constituyeron la muestra.

19.Distribución de los trabajos de grado según el género de los participantes.

20.Distribución de los trabajos de grado según el número de referencias reportadas.

Castera etal. (2008) identificaron para el primer aspecto una serie de indicadores, los cuales se exponen de la siguiente manera: los índices cuantitativos útiles para medir, entre otros, 
los niveles de producción científica de un país, una institución, un autor o una publicación. En ellos influyen muchas y muy variadas circunstancias. Estas medidas sirven para comparar las producciones científicas entre grupos similares, pero no para evaluar la calidad de la producción de un país, una institución o una publicación. Estos indicadores permiten que se realice un análisis más específico y organizado de los documentos obtenidos. Se analizan diferentes elementos que se encuentran en común en las creaciones literarias, lo que permite cuantificar la cantidad de producción que se realiza a nivel nacional y entre universidades. Esto permite además ver el desarrollo y la importancia que se le da a estos trabajos y al tema investigado.

\subsection{Procedimiento}

En primer lugar se identificó en la página de Ascofapsi las facultades de psicología adscritas. Esto con el fin de iniciar la búsqueda de los trabajos de grado (pregrado y posgrado) de cada una de las universidades. Esta primera búsqueda dio como resultado un listado de once universidades.

A continuación, se consultó en las bases de datos de dichas universidades a través del catálogo en línea de sus respectivas bibliotecas. Tras la consulta de estos trabajos de grado se revisó el título y las palabras clave. Esto con el fin de verificar si tenían la palabra liderazgo en alguna de estas partes y si eran trabajos de grado realizados entre los años 2000 y 2011, ya que estos eran los filtros para seleccionar las tesis.

Como resultado de búsqueda se identificaron en total 52 trabajos de grado de pregrado y posgrado que abordaban el tema de liderazgo. Sin embargo, solo 22 reunían los criterios dados por los filtros. Se eliminaron también cinco universidades, tales como el Rosario, la universidad Nacional, el Externado y la Konrad Lorenz, en las cuales no se logró encontrar ningún trabajo de grado que reuniese los criterios de selección dados por los filtros planteados inicialmente.

También es importante mencionar que de la universidad de la Sabana no se logró estudiar ningún trabajo de grado. Esto debido a la pérdida que hubo de estos por la pasada ola invernal, en la que ellos quedaron sumergidos y no se logró recuperarlos, en particular, los relativos al período que abarca la investigación.

Posteriormente, se recolectó la información en el formato de registro que se utilizó para organizar dichos 
trabajos y se procedió a analizar las variables a través de la estadística descriptiva y el análisis lexicométrico. Esto permitió obtener los resultados que serán explicados en el apartado 3 .

\subsection{Consideraciones éticas}

Teniendo en cuenta los lineamientos de la resolución No. 008430 de 1993 del Ministerio de Salud, por la cual se establecen las normas científicas, técnicas y administrativas para la investigación en salud, la presente investigación resalta, según el artículo 11 del capítulo II, que el presente estudio es una investigación sin riesgo, debido a que no se realiza ninguna intervención o modificación intencionada de las variables biológicas, fisiológicas, psicológicas o sociales de los individuos que participan en el estudio. Igualmente, en el desarrollo de este estudio bibliométrico, se hace un análisis cuidadoso del contenido de la producción científica consultada, manteniendo la veracidad y reserva de la información y garantizando los derechos de autor de las fuentes revisadas.

La ley 1090 del psicólogo (de 2006), también establece el código deontológico y bioético, así:

El presente Código Deontológico y Bioético, estádestinadoa servircomo regla de conducta profesional en el ejercicio de la Psicología en cualquiera de sus modalidades, proporcionando principios generales que ayuden a tomar decisiones informadas en la mayor parte de las situaciones con las cuales se enfrenta el profesional de la Psicología, fundamentado en los principios de beneficencia, no maleficencia, autonomía, justicia, veracidad, solidaridad, lealtad y fidelidad además de las contempladas en la presente ley (p. 10).

Es importante valorar la dimensión ética de las investigaciones con base en diferentes criterios. Emanuel y Lolas(2003, citados en Rendón, 2008) proponen: los méritos técnicos, en la medida en que el estudio se encuentre bien planteado, en el sentido de insertarse de manera coherente y rigurosa en una tradición de pensamiento; el mérito científico, ya que la investigación contribuye a una mejor comprensión del mundo y del estado de conocimiento sobre ciertas cuestiones, y el mérito social, que alude a la legitimidad con que se conduce el proyecto, los efectos saludables que tiene su realización, la justicia que promueve, su aporte al logro de metas sociales, el uso responsable de recursos y la solución ecuánime de los conflictos de intereses.

Esta investigación bibliométrica contempla los principios éticos y el 
código deontológico de los profesionales de Psicología para la revisión y análisis del material proporcionado por las universidades. Esto con el fin de convertirse en un material que aporte y beneficie a la comunidad científica y que cumpla los estándares de planteamiento, coherencia y rigurosidad para ser considerada como investigación científica.

\section{RESULTADOS}

Acontinuación, se describen algunos de los indicadores que se analizaron en los veintidós trabajos de grado encontrados en las facultades de psicología de la ciudad de Bogotá adscritas a Ascofapsi, relacionados con la temática de liderazgo (ver tabla 1). Los demás indicadores serán analizados por medio del programa Spad.

Tabla 1. Trabajos de grado revisados por universidades

\begin{tabular}{|l|c|c|}
\hline \multicolumn{1}{|c|}{ Universidades } & Total & $\%$ \\
\hline $\begin{array}{l}\text { Universidad Católica de } \\
\text { Colombia }\end{array}$ & 8 & 36 \\
\hline Universidad Piloto & 5 & 23 \\
\hline Universidad Pontificia Javeriana & 3 & 14 \\
\hline $\begin{array}{l}\text { Institución Universitaria Los } \\
\text { Libertadores }\end{array}$ & 3 & 14 \\
\hline Universidad Incca de Colombia & 1 & 5 \\
\hline Universidad de Los Andes & 1 & 5 \\
\hline Universidad Santo Tomás & 1 & 5 \\
\hline Total & & 100 \\
\hline
\end{tabular}

Fuente: elaboración propia.
Los registros que se tuvieron en cuenta para realizar el análisis bibliométrico fueron: trabajos de grado por año, filiación institucional, tipo de documento, enfoque, tipo de estudio, temática relacionada, instrumentos, número de participantes y referencias.

En relación con la elaboración de los trabajos de grado por año, se encontró que los dos años en los que se elaboraron más trabajos de grado fueron 2002 y 2010. Según la filiación institucional, predominan las Universidades Católica y Piloto, con $36 \%$ y $23 \%$ del total de la muestra, respectivamente.

Según el tipo de documento, los trabajos se distribuyen en empíricos con un $68 \%$ y teóricos $32 \%$. Entre tanto, el enfoque de investigación que predomina es el cuantitativo (77\%), 9 trabajos correspondiendo a estudios descriptivos (53\%), 6 a exploratorios $(35 \%)$ y 2 a correlacionales (35\%). Por otra parte, el 32\% se inscribe en el enfoque cuantitativo, en donde se evidenció la realización de cuatro estudios de caso $(80 \%)$ y uno de tipo hermenéutico.

Las temáticas que los investigadores relacionan más con el tema de liderazgo son: medición (45\%), clima organizacional $(23 \%)$, modelos de liderazgo (13\%), desempeño laboral 
(9\%), género y estado del arte, cada uno con un $5 \%$.

En relación con los instrumentos que se utilizan en los trabajos de grado, se encontró que once corresponden a otros $(52 \%)$, seis a cuestionarios (29\%) y cuatro a encuestas $(19 \%)$, dentro del enfoque cuantitativo, mientras que en el enfoque cuantitativo se identificaron dos en otros ( $40 \%$ ) y cuadernos de campo, historias de vida y entrevistas abiertas, cada una con un $20 \%$. De acuerdo con la muestra utilizada para las investigaciones, se halla que nueve corresponden a 30 o menos participantes, cuatro entre 31 y 100 , dos entre 101 y 200 y tres entre 201 y 300 . Según el género de los participantes, el $86 \%$ corresponde a una muestra de composición mixta (hombres y mujeres). En cuanto al número de referencias utilizadas se evidencia que el $55 \%$ corresponde a libros, seguido por otros con un $19 \%$, revistas $14 \%$, $9 \%$ medios electrónicos y $3 \%$ tesis.

A continuación, se presenta la segunda fase del análisis, relativa a los resultados del análisis lexicométrico de los siguientes indicadores, a saber: resumen, objetivo general, objetivos específicos, pregunta problema y definición del autor.

La tabla 2, señala el análisis por relevancia de palabras encontradas en el instrumento utilizado para la recolección de información de cada una de las unidades de análisis.

Tabla 2. Número de frecuencia de palabras

\begin{tabular}{|l|c|c|}
\hline \multicolumn{1}{|c|}{ Palabras } & Frecuencia & Porcentaje \\
\hline Liderazgo & 118 & $2,17 \%$ \\
\hline Competencias & 35 & $0,64 \%$ \\
\hline Trabajo & 27 & $0,49 \%$ \\
\hline Proceso & 26 & $0,47 \%$ \\
\hline Líderes & 26 & $0,47 \%$ \\
\hline Nivel & 24 & $0,44 \%$ \\
\hline Equipo & 24 & $0,44 \%$ \\
\hline
\end{tabular}

Fuente: elaboración propia.

La primera tendencia, identificada mediante el análisis de las palabras más frecuentes, agrupa palabras que representan procesos asociados directamente con el tema de investigación, los cuales tienen una gran incidencia en la ejecución del líder en la organización. Dentro de estas se encontraron: liderazgo, competencias, trabajo, proceso, líderes. Esto, haciendo referencia a las funciones que se buscan y deben ejercer los líderes en los contextos organizacionales, también enmarcados como principal característica de esta investigación. La segunda tendencia expone palabras que se refieren directamente a la relación que tiene el líder con grupos y equipos, los cuales ayudan a jalonar procesos al interior de la organización. 
Otra tendencia es la forma de medir y evaluar el tema del liderazgo en su ejercicio diario, dentro de estas categorías aparecieron con mayor frecuencia palabras como evaluar, empresa, variables relación e instrumento.

Como otra tendencia temática, se identificaron palabras que proporcionan una definición y caracterización del proceso de ser líder en el ámbito organizacional, es decir, palabras que aportan una definición en materia teórica al concepto. Estas son: competencias, equipo, empresa y proceso.

Por último, se presenta la tendencia de aquellas palabras que se refieren al contexto o ambiente en el cual se desarrolla el tema de liderazgo. Esta categoría sitúa el avance del tema en contextos específicos, presentando palabras que hacen evidente la participación de los individuos en los procesos dentro de las organizaciones. Las palabras halladas son: empresa, trabajo y nivel. Posteriormente, se enmarcan palabras con tendencias hacia los grupos y los equipos, que hacen parte fundamental en el proceso de desarrollo del líder.

A continuación, en la tabla 3 se indica la relevancia de los segmentos de palabras más frecuentemente halladas por medio del análisis lexicométrico.
Tabla 3. Segmentos de palabras frecuentes

\begin{tabular}{|l|c|c|}
\hline \multicolumn{1}{|c|}{ Palabras } & Frecuencia & Porcentaje \\
\hline Trabajo en equipo & 108 & $1,99 \%$ \\
\hline Sobre liderazgo & 105 & $1,93 \%$ \\
\hline Por competencia & 92 & $1,69 \%$ \\
\hline Los líderes dirigen & 86 & $1,58 \%$ \\
\hline Los jefes lideran & 85 & $1,56 \%$ \\
\hline Los indicadores & 84 & $1,54 \%$ \\
\hline Liderazgo en los & 80 & $1,47 \%$ \\
\hline Liderazgo de & 77 & $1,41 \%$ \\
\hline $\begin{array}{l}\text { Las habilidades } \\
\text { sociales }\end{array}$ & 76 & $1,40 \%$ \\
\hline Las organizaciones & 73 & $1,34 \%$ \\
\hline $\begin{array}{l}\text { La competencia de } \\
\text { liderazgo }\end{array}$ & 67 & $1,23 \%$ \\
\hline La empresa & 62 & $1,14 \%$ \\
\hline Estilo de & 52 & $0,95 \%$ \\
\hline El desempeño & 35 & $0,64 \%$ \\
\hline El desarrollo & 34 & $0,62 \%$ \\
\hline $\begin{array}{l}\text { De la inteligencia } \\
\text { emocional }\end{array}$ & 30 & $0,55 \%$ \\
\hline De los líderes & 28 & $0,51 \%$ \\
\hline $\begin{array}{l}\text { Competencia de } \\
\text { liderazgo }\end{array}$ & 4 & $0,07 \%$ \\
\hline $\begin{array}{l}\text { Clima } \\
\text { organizacional }\end{array}$ & 2 & $0,03 \%$ \\
\hline Fing & & \\
\hline
\end{tabular}

Fuente: elaboración propia.

De acuerdo con lo anterior, se puede decir que la mayor frecuencia de segmentos, representado 108 veces en el instrumento de recolección de información de las unidades de análisis, 
está en el constructo de trabajo en equipo, seguido por la temática principal de análisis y de factores importantes, tales como las habilidades sociales, las organizaciones, los indicadores y la capacidad. Estos elementos tienden a mostrar la importancia en la manera como el liderazgo es percibido.

Una segunda tendencia, que conviene tener en cuenta, se encuentra en las habilidades que debe tener el líder para generar en sus subordinados competencias que sean acordes a las labores que estén desarrollando. Esto con el fin de no tener un estancamiento y lograr obtener conocimientos alrededor de otras áreas de interés afines a los estudios e investigaciones que estos realizan.

El líder se identifica como alguien propositivo, con capacidad para conseguir en sus empleados el desarrollo y uso de herramientas propias que lo inviten a crear relaciones que sean propicias para el trabajo en equipo, en beneficio de su área. Los segmentos arrojados fueron: capacidad para, desarrollo, empleados, la relación, el desempeño, laconductadecompartir conocimiento y el trabajo en equipo.

Finalmente, estos segmentos repetitivos dan cuenta, por medio de la medición y análisis de las palabras, de la importancia de las caracterís- ticas fundamentales que permiten que el líder ejerza su rol, que estos aspectos deben ser tenidos en cuenta en el contexto organizacional. Con esto se invita a conocer los trabajos que han sido efectuados en relación con la manera en la que este debe manejar su equipo de trabajo.

La figura 1 muestra los resultados del análisis factorial arrojado por el paquete estadístico SPAD. Estos permiten describir algunas tendencias importantes encontradas en el estudio bibliométrico realizado. Es importante tener en cuenta que, de acuerdo con los hallazgos, se denominó al eje $\mathrm{X}$ del grafico tipos de muestreo y al eje Y fuente de consulta. Cuando en el programa SPAD la varianza explicada del análisis de correspondencia es mayor al $75 \%$, esto significa que su poder de explicación es muy elevado, ya que entre mayor porcentaje tenga este, puede considerarse más verdadera y confiable la explicación. La varianza explicada del análisis de correspondencias fue de $77,62 \%$, lo que significa que los resultados del plano factorial son verdaderos y confiables para su interpretación.

Los datos procesados por el programa SPAD en los planos factoriales señalan que en el primer factor hubo una varianza explicada del 40,76\%, correspondiente al eje $\mathrm{X}$ del plano 
factorial y que, en el segundo factor, hubo una del $36,86 \%$, en relación con el eje Y. En total se encontró una varianza explicada del análisis de correspondencias del 77,62\%.

Los porcentajes corresponden a la varianza explicada de los planos factoriales. Estos tienen que ver con el grado de relevancia de la explicación que tienen las representaciones gráficas de los planos. Estos simbolizan qué tan importantes y repre- sentativas son las interpretaciones que se obtuvieron de los planos factoriales. Los fenómenos agrupados en el eje $\mathrm{X}$ obedecen generalmente a la variable independiente. Entonces, se considera que son los fenómenos que podrían ser los factores asociados al eje Y como causa probable. Esto, en la interpretación, puede considerarse para referenciar que dadas las circunstancias o factores descritos en $\mathrm{X}$ es susceptible que se produzcan los factores asociados a Y.

Figura 1. Resultados del análisis factorial

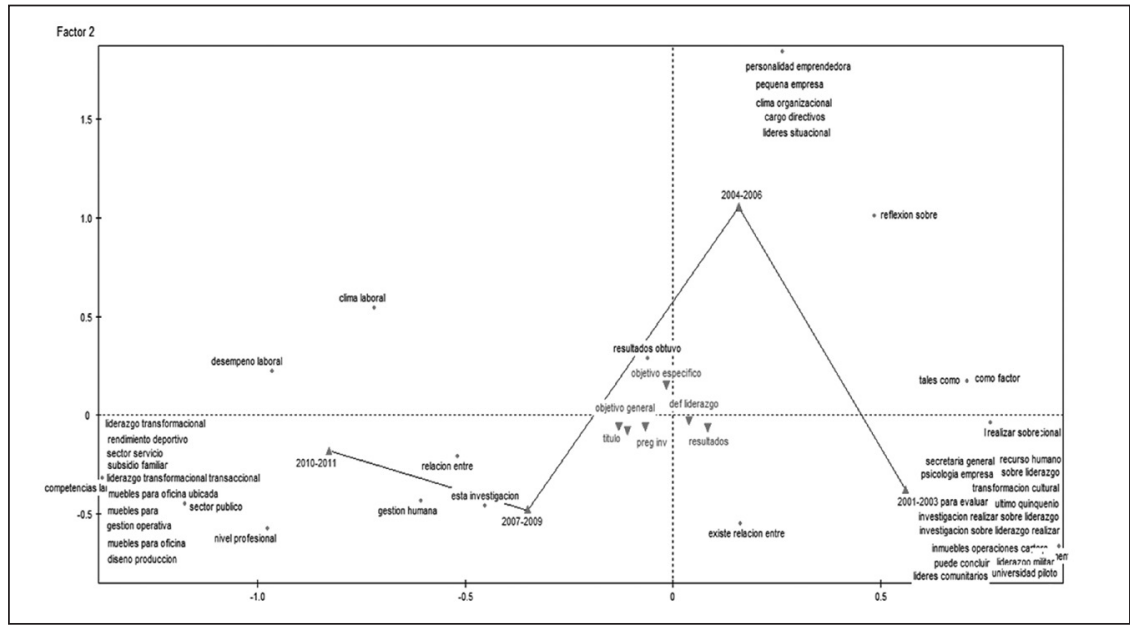

Fuente: elaboración propia.

En la figura 1 se destaca el hecho de que los trabajos de grado elaborados entre 2001 y 2003 se centraron en estudiar la influencia del liderazgo en las áreas de la gestión humana, realizando investigaciones sobre el liderazgo en asociación con la cul- tura de las empresas. Esto en contextos muy variados, que van desde líderes comunitarios hasta organizaciones militares.

Entre 2004 y 2006, los trabajos de grado tendieron a realizar investiga- 
ciones en pequeñas empresas sobre el liderazgo situacional. En estos el líder expone sus capacidades para diagnosticar las condiciones del contexto en el que se encuentra, para poder crear combinaciones adecuadas de los tipos de conducta que deben ser desempeñados de acuerdo al medio predominante en los cargos directivos (Sánchez, 2000). Esto relacionándolo con los rasgos de la personalidad emprendedora, los cuales deben caracterizar a cualquier líder, sin importar el entorno.

Entre 2007 y 2011 se encontró que las unidades de análisis se enfocaron en el estudio de los modelos de liderazgo transformacional y transaccional, tanto en empresas privadas como públicas, eincluso en entidades deportivas distritales como el IDRD (Instituto Distrital de Recreación y Deporte). En este caso, se concentraron en la manera en la que este modelo de liderazgo influencia el rendimiento deportivo, esto en el IDRD y en las áreas de gestión humana de las organizaciones en general.

Es posible afirmar que los trabajos de grado estudiados entre 2000 y 2011 demuestran que las definiciones de liderazgo encontradas tienden a describirlo como un proceso, es decir, como algo que se ejerce a lo largo del tiempo. Un proceso consistente en el hecho de que una persona use su influencia sobre otras. Esto con el fin de estructurar y conducir las funciones y relaciones dentro de un grupo u organización.

\section{DISCUSIÓN Y CONCLUSIONES}

De acuerdo con los hallazgos encontrados a través del estudio bibliométrico de liderazgo, se puede corroborar que la temática es abordada desde diferentes disciplinas, diferentes a la psicología, las cuales están realizando aportes significativos.

Desde la antropología, por ejemplo, el liderazgo está siendo visto como la habilidad que tiene un sujeto para promocionar y proteger los valores institucionales, así como para ser un elemento motivador que busca mantener e incrementar la unidad de una organización (Caparás \& Chinchilla, 2000).

Con respecto a disciplinas como la sociología, se ha manejado la temática dando importancia al género, a la vinculación de la mujer en el mundo laboral y cómo los cambios sociales de las últimas décadas han influido en el crecimiento de ella al interior de las organizaciones, hasta lograr desempeñar cargos que implican liderazgo. Estos, en el pasado, eran exclusivos para los hombres (Bernal, 1998). El derecho, por su 
parte, se ha centrado más en velar por la igualdad de derechos y oportunidades laborales entre hombres y mujeres, para desempeñar cargos directivos dentro de las organizaciones (OIT, 2010).

En la administración se habla de varias teorías del liderazgo situacional, teniendo en cuenta la relevancia de los elementos de la situación. Desde esta teoría, se menciona que la efectividad del liderazgo depende de la capacidad que tiene el líder para diagnosticar las condiciones de una situación y reaccionar con una combinación adecuada de ciertos tipos de conducta, a la vez que perciben como apropiados algunos ajustes entre el liderazgo y la situación (Sánchez, 2000).

Desde la perspectiva psicológica, el liderazgo es observado como una política fundamental en las organizaciones, haciendo referencia en particular a las áreas de los recursos humanos. Esta es vista como un conjunto de personas multidisciplinares que tratan de integrar a los empleados en un proyecto empresarial del cual hacen parte (Gómez, 2008).

El constructo del liderazgo guarda relación con diferentes aspectos psicológicos como son el trabajo en equipo (Mendoza, 2008; Sánchez, Tapia, \& Becerra, 2006), la comu- nicación (García, 2012), la motivación (Castro, 2006), las habilidades (Benatuil \& Castro, 2007), la personalidad (Godoy \& Mladinic, 2009) y las actitudes (Benatuil \& Castro, 2007). Estos aspectos denotan que se puede realizar una mirada holística de liderazgo, entendiendo que ejercerlo implica una gran variedad de aspectos propios del ser humano, los cuales se relacionan con determinantes como el clima organizacional, la cultura (Godoy \& Mladinic, 2009) y el desempeño (Salido, 2001), que no solo se evidenciaron a partir del marco disciplinar, sino en la revisión de los diferentes indicadores que permitieron recuperar las temáticas de cada una de las unidades de análisis seleccionadas. Estos aspectos también fueron corroborados gracias al análisis realizado con el paquete estadístico SPAD.

En cuanto a los tipos de investigación que se han realizado al abordar el tema de liderazgo, ha existido una tendencia a estudiar la temática mediante estudios empíricos con enfoque cuantitativo, en su mayoría de tipo descriptivo, que permiten arrojar datos estadísticos acerca de muestras mixtas (de hombres y mujeres). Se han aplicado cuestionarios, por ejemplo, en la medida en que estos indagan con mayor profundidad los conocimientos, actividades y prácticas que caracterizan al líder en su 
entorno o ante situaciones que retan su capacidad para liderar.

En la revisión realizada se encontraron varios instrumentos que tuvieron como propósito realizar diferentes mediciones sobre el tema. Algunos de estos son: 1) medición de liderazgo transformacional y transaccional (Bass \& Avolio, 1992), 2) Leader Effectiveness and Adaptability Description (LEAD) (Sánchez, 2000), 3) Escala de Evaluación del Líder Deportivo (EELD) (Arce, Torrado, Andrade, Garrido, \& De Francisco, 2008), 4) atributos del líder (Hanges \& Dickson, 2004 citados en Castro, 2006), y 5) Bem Sex Role Inventory (BSRI), creado por Bem (1974 citado en Cuadrado, 2004).

Conviene resaltar la importancia de cada uno de los instrumentos mencionados. Estos se hacen con el fin de medir estilos de liderazgo y evidenciar la manera en la que estos pueden ser aplicados según el contexto. Esto teniendo en cuenta también otros factores importantes para la ejecución del líder. Así mismo, cabe resaltar que para aplicar alguno de estos es fundamental revisar detalladamente los aspectos que se tienen en cuenta y la adaptabilidad a contextos como el colombiano, por ejemplo, así como la particularidad de la empresa en donde este vaya a ser implementado.
De otro lado, se considera que es importante no dejar de lado las investigaciones cualitativas que también han abordado el tema. Estas han realizado un amplio abordaje teórico acerca del constructo, llevando a cabo estudios de caso, en su mayoría. Este tipo de trabajo se destaca debido a que el investigador se vuelve participe del grupo que está siendo estudiado, en la medida en que asume como escenario de labor de campo su propio lugar de trabajo, en donde tiene mayor oportunidad de interactuar con compañeros y cuenta con la experiencia cotidiana de vivir diferentes formas de liderazgo.

En cuanto a los instrumentos utilizados en este tipo de enfoque, las fichas descriptivas, que se ubicaron en la categoría de otros, fueron utilizadas como registro de información en los diferentes contextos estudiados. Estas dan lugar a la descripción de cada uno de los sitios, con el fin de registrar, analizar, entender y justificar la información que había sido obtenida del grupo estudiado. Esto proporciona un registro de información resumido y organizado, clasificando los datos en un formato de fácil diligenciamiento y posterior comprensión. Es también una herramienta práctica, ya que el investigador se encuentra inmerso dentro del contexto y es participante del mismo. Vale la pena aclarar que los resul- 
tados que se están mencionando, de acuerdo con el presente estudio bibliométrico, representan en su mayoría investigaciones realizadas en la ciudad de Bogotá, encontradas en diferentes bibliotecas de las universidades.

A través del estudio bibliométrico, se pudo identificar también que existe una predominancia significativa en cuanto a los textos de consulta, que en su gran mayoría corresponden a libros y revistas, tanto de psicología como de otras disciplinas, siendo estas fuentes de investigación que abarcan un amplio contenido de la temática. Sin embargo, llama la atención el hecho de que la fuente de página web más utilizada sea el portal Monografías.com. Esta es una fuente de fácil acceso, pero que no cumple las características de indexación que se requieren a la hora de determinar fuentes primarias en un documento. Este aspecto hace que los textos realizados por los estudiantes puedan llegar a carecer del sustento y la profundidad requeridos tanto para el nivel de pregrado como de posgrado.

Mediante el análisis cuantitativo de textos, se identificó una mayor productividad en el tema en los años 2002, 2006 y 2010. Esto indica una tendencia particular marcada a través de los años. Por otra parte, se establece también una tendencia en cuanto al tipo de liderazgo ejercido por los líderes en las organizaciones, el cual hace énfasis en estilos desde el situacional, reflejado en el trabajo de Hersey y Blanchard (1982), hasta el transaccional-transformacional, incorporado en la obra de Bass y Avolio (Bass, Avolio, Jung, \& Berson, 2003). Estos son utilizados de acuerdo con la situación que se presente en la organización y, de este modo, el líder asume cualquiera de ellos, sin que uno sea considerado necesariamente mejor que el otro (García, 2012).

Finalmente, se puede decir que la presente investigación bibliométrica permitió evidenciar nuevamente que el liderazgo no solo tiene aportes hacia el contexto laboral, es decir, hacia la organización en cuanto tal, sino que también genera un crecimiento personal que fortalece el desarrollo integral del individuo, en el contexto en el generalmente permanece la mayor parte de su tiempo.

\section{REFERENCIAS}

Aguilar, M. C., López, W., Barreto, I., Rey, Z., \& Vargas, E. (2007). Análisis bibliométrico de los trabajos de grado del área organizacional de la Facultad de Psicología de la Universidad Santo Tomás. Diversitas Perspectivas en Psicología, 3(2), 317-334. 
Arce, R., Torrado, J., Andrade, E., Garrido, J., \& De Francisco, C. (2008). Elaboración de una escala para la medida de la capacidad de liderazgo de los deportistas de equipo. Revista Psicothema, 2(3), 913-917.

Barreto, I., Borja, H., Sabucedo, J., López, W., \& Alzate. M. (2010). Creencias legitimadoras y deslegitimadoras difundidas por la prensa española sobre el asesinato político. Revista Latinoamericana de Psicología, 3(2), 437-452.

Bass, B. M(1999). Two decades of research and development in transformational leadership. European Journal of Work and Organizational Psychology, 8(1), 9-13.

Bass, B. M., \& Avolio, B. J. (1992). Organizational description questionnaire. Red-wood City: Mind Garden.

Bass, B. M., \& Avolio, B. J. (1994). Shatter the glass ceiling: Women may make better managers. Human Resource Management, 33(4), 549-560.

Bass, B. M., \& Avolio, B. J. (2000). MLQ Multifactor Leadership Questionnaire.

Bass, B., Avolio, B., Jung, B., \& Berson, Y. (2003). Predicting unit performance by assessing transformational and transactional leadership. Journal of Applied Psychology, 88(2), 207-218.

Benatuil, D., \& Castro, A. (2007). Estilos de liderazgo, inteligencia y conocimiento tácito. Anales de Psicología, 23(5), 216-225.

Bernal, A. (1998). Movimientos feministas y cristianismo. Madrid: Rialp.

Bordones, M., \& Zulueta, M. A. (1999). Evaluación de la actividad científica a través de indicadores bibliométrico. Revista española de cardiología, 52(10) 790-800.

Camps, D. (2007). Estudio bibliométrico general de colaboración y consumo de la información en artículos originales de la revista Universitas Médica, período del 2002 al 2006. Universitas Médica, 48(4), 358-365.

Caparás, V., \& Chinchilla, N. (2000). Teoríainstitucionalyteoría antropológica del liderazgo: salvando un vacio de 40 años [documento de investigación No 407]. Barcelona: Universidad de Navarra.

Capowski G. (1994). Anatomy of a leader: where are the leaders of tomorrow? Management Review, 83, 10-17.

Cardona, J. (2004). ¿Qué modelo de liderazgo utilizar en la formación y desarrollo de directivos? Revista Intangible Capital, 8(1), 3-6.

Castera, V. T., Sanz Valero, J., Juan Quilis, V., Wanden Berghe, C., Culebras, J. M., García de Lorenzo y Mateos, A., \& Grupo de Comunicación y Documentación Científica en Nutrición CDCNut SENPE. España. (2008). Es- 
tudio bibliométrico de la revista Nutrición Hospitalaria en el periodo 2001 a 2005: parte 2, análisis de consumo; referencias bibliográficas. Nutrición Hospitalaria, 23(6), 541-546.

Castro, A. (2006). Teorías implícitas de liderazgo, contexto y capacidad de conducción. Anales de Psicología, 22(1), 89-97.

Cendrós, J., Rincón, S., \& Ugas, L (2004). Estilos de liderazgo y escala de cambio en los medios de comunicación social. Revista venezolana de ciencias sociales, 8(2), 225-239.

Cruz, J., \& Javela, L. (2004). Una aproximación al paradigma dominante de la psicología social a partir de una muestra de los manuales publicados desde el año 2000. Acta colombiana de psicología, 12(4), 39-58.

Cuadrado, I. (2004). Valores y rasgos estereotípicos de género de mujeres líderes. Revista Psicothema, 16(4), 270-275.

Eagly, A., \& Karau, S. (2002). Role congruity theory of prejudice toward female leaders. Revista Psicológica, 109(3), 573-598.

Eden, D. (1992). Leadership and expectations: Pygmalion effects and other self-fulfilling prophecies in organizations. Leadership Quarterly, 3, 271-335.

Eisenbach, R., Watson, K., \& Pillai, R. (1999). Transformatio- nal leadership in the context of organizational change. Journal of organizational change management. 12(2), 80-93.

Fleishman, E. A., Mumford, M. D., Zaccaro, S. J., Levin, K. Y., Korotkin, A. L., \& Hein, M. B. (1991). Taxonomic efforts in the description of leader behavior: A synthesis and functional interpretation. Leadership Quarterly, 4, 245287.

García, M. (2012). Liderazgo transformacional y aceptación del cambio organizacional. Londres: Académica Española.

Godoy, L., \& Mladinic, A. (2009). Estereotipos y roles de género en la evaluación laboral y personal de hombres y mujeres en cargos de dirección. Revista Psykhe, 18(2), 51-64.

Gómez, R. (2008). El liderazgo empresarial para la innovación tecnológica en las micro, pequeñas y medianas empresas. Pensamiento y Gestión, 24(2), 62-91.

Hater, J. J., \& Bass, B. M. (1988). Superiors' evaluations and subordinates' perceptions of transformational and transactional leadership. Journal of Applied Psychology, 73, 695-702.

Helland, T., Wazeter, D., \& Bayazit, M. (2009). Union leadership and member attitudes: A multilevel analysis. Journal of Applied Psychology, 94(5), 392-410. 
Hersey, P. \& Blanchard, K.H. (1982). Leadership style: attitudes and behavior. Training and Development Journal, 36(2), 50-52

Hernández, R., Fernández, C., \& Baptista, P. (2006). Metodología de la investigación. México: McGraw-Hill.

Hinkin, T., \& Tracey, B. (1994), Transformational leadership in the hospitality industry. Journal of Hospitality y Tourism Research, 18(1), 49-63.

Hollander, E. P., \& Julian, J. W. (1969). Contemporary trends in the analysis of leadership processes. Psychological Bulletin, 71, 387-397.

House, R. J., \& Podsakoff, P. M. (1994). Leadership effectiveness: Past perspectives and future directions for research. En J. Greenberg (Ed.), Organizational Behavior: The State of the Science (pp. 45-82). Hillsdale: Erlbaum.

House, R. J., \& Shamir, B. (1993). Toward the integration of transformational, charismatic, and visionary theories. En M. Chemers \& R. Ayman (Eds.), Leadership theory and research: Perspectives and directions (pp. 81-107). New York: Academic Press.

House, R. J., Javidan, M., Hanges, P. J., \& Dorfman, P. W. (2002). Understanding cultures and implicit leadership theories across the globe: An introduction to pro- ject GLOBE. Journal of World Business, 37(1), 3-10.

House, R., Wright, N., \& Aditya, R. (1997). Cross cultural research on organizational leadership: A critical analysis and a proposed theory. News Perspective in International Industrial Organizational Psychology, 1(2), 535-625. Lebart, L., Salem, A., \& Bécue, M. (2000). Análisis estadístico de textos. Madrid: Editorial Milenio.

Ley 1090 del Psicólogo (2006). Código deontológico y bioético y otras disposiciones. Diario oficial: Edición 46.383.

Massi, R. J., \& Cooke, R. A. (2000). Effects of transformational leadership on subordinate motivation, empowering norms, and organizational productivity. The International Journal of Organizational Analysis, 8(1), 16-47.

Mendoza, I. A., Ortiz, M. F., \& Parker, H. C. (2007). Dos décadas de investigación y desarrollo en liderazgo transformacional. Revista Centro de Investigación. Universidad de la Salle, 7(27), 25-41.

Mendoza, M. (2008). Aproximación al liderazgo desde la caracterización de redes sociales presentes en el aula. Revista Facultad de Ciencias Económicas, 16(2), 141-160.

Mendoza, M. R., \& Ortiz, C. (2006). El liderazgo transformacional, 
dimensiones e impacto en la cultura organizacional y eficacia de las empresas. Revista facultad de ciencias económicas, 14(1), 118134.

Meneses, A., Calle, A., \& García, M. (2008). Análisis bibliométricodel abordaje psicológico de la enfermedad cardiovascular de enero de 1998 a diciembre de 2007. Especialización en Psicología Médica y de la Salud, trabajo de grado inédito, Universidad El Bosque, Bogotá, Colombia.

Naranjo, C. (2001). El liderazgo en la gestión del conocimiento. Revista Perspectiva Psicológica, Biblioteca virtual en psicología, 10(3), 91-117.

Organización Internacional del Trabajo (OIT). (2010). Primer informe global sobre discriminación en el trabajo. Recuperado el 10 de septiembre de 2011, de http:// www.oit.org.pe/index.php?option $=$ com content\&view $=$ article $\& \mathrm{i}$ ed $=306 \% 3$ Ala-oit-lanza-el-primer-informe-global-sobre-discriminacion.

Paglis, L., \& Green, S. (2002). Leadership self-efficacy and managers' motivation for leading change. Journal of organizational behavior, 23(2), 215-235.

Pedraja, L., \& Rodríguez, E. (2004). Efectos del estilo de liderazgo sobre la eficacia de las organizaciones públicas. Revista Facultad de Ingeniería, 12(1), 63-73.
Pedraja, L., Rodríguez, E., \& Rodríguez, J. (2006). Liderazgo y decisiones estratégicas: una perspectiva integradora. Revista Interciencia, 31(3), 587-582.

Rendón, M. (2008). Elementos a tener en cuenta para consideraciones éticas en investigación con participantes humanos. Trabajo inédito. Bogotá: Facultad de Psicología, Universidad Santo Tomás.

Salido, O. (2001). La movilidad ocupacional de las mujeres en España. Por una sociología de la movilidad femenina. Madrid: Centro de Investigaciones Sociológicas.

Sánchez, E. (2000). Teoría del liderazgo situacional en la administración local: validez del modelo. Revista Psicothema, 12(3), 435439.

Sánchez, V., Tapia, C., \& Becerra, S. (2006). Liderazgo: una mirada desde directivos, profesores y alumnos. Revista de Psicología, 15(2) 29-52.

Schein, V. (2001). A global look at psychological barriers to women's progress in management. Journal of Social Issues, 57(1), 675-688.

Yukl, G. (1989). Managerial leadership: A review of theory and research. Journal of Management, 15, 251-289.

Yukl, G. (2008). Managerial leadership: A review of theory and 
research. Journal of Management, 15(2), 251-289.

Yukl, G., \& Van Fleet, D. (1992) Theory and research on leadership in organizations. En M. D. Dunnette \& L. M. Hoveh (Eds.) (1992). Handbook of industrial and organizational psychology
(Vol. 3, pp. 147-197). Palo Alto: Consulting Psychologists Press. Yukl, G., \& Van Fleet, D. D. (1982). Cross situational multimethod research on military leader effectiveness. Organizational Behavior and Human Performance, 30, 87-108. 